\title{
Comparative transcriptome sequencing of tolerant rice introgression line and its parents in response to drought stress
}

Liyu Huang ${ }^{1+}$, Fan Zhang ${ }^{1+}$, Fan Zhang ${ }^{1,2}$, Wensheng Wang ${ }^{1}$, Yongli Zhou ${ }^{1 *}$, Binying Fu ${ }^{1 *}$ and Zhikang Li ${ }^{1}$

\begin{abstract}
Background: Rice (Oryza sativa. L) is more sensitive to drought stress than other cereals, and large genotypic variation in drought tolerance (DT) exists within the cultivated rice gene pool and its wild relatives. Selective introgression of DT donor segments into a drought-sensitive (DS) elite recurrent parent by backcrossing is an effective way to improve drought stress tolerance in rice. To dissect the molecular mechanisms underlying DT in rice, deep transcriptome sequencing was used to investigate transcriptome differences among a DT introgression line H471, the DT donor P28, and the drought-sensitive, recurrent parent HHZ under drought stress.

Results: The results revealed constitutively differential gene expression before stress and distinct global transcriptome reprogramming among the three genotypes under a time series of drought stress, consistent with their different genotypes and DT phenotypes. A set of genes with higher basal expression in both H471 and P28 compared with $\mathrm{HHZ}$ were functionally enriched in oxidoreductase and lyase activities, implying their positive role in intrinsic DT. Gene Ontology analysis indicated that common up-regulated genes in all three genotypes under mild drought stress were enriched in signaling transduction and transcription regulation. Meanwhile, diverse functional categories were characterized for the commonly drought-induced genes in response to severe drought stress. Further comparative transcriptome analysis between $\mathrm{H} 471$ and $\mathrm{HHZ}$ under drought stress found that introgression caused wide-range gene expression changes; most of the differentially expressed genes (DEGs) in $\mathrm{H} 471$ relative to $\mathrm{HHZ}$ under drought were beyond the identified introgressed regions, implying that introgression resulted in novel changes in expression. Co-expression analysis of these DEGs represented a complex regulatory network, including the jasmonic acid and gibberellin pathway, involved in drought stress tolerance in $\mathrm{H} 471$.

Conclusions: Comprehensive gene expression profiles revealed that genotype-specific drought induced genes and genes with higher expression in the DT genotype under normal and drought conditions contribute jointly to DT improvement. The molecular genetic pathways of drought stress tolerance uncovered in this study, as well as the DEGs co-localized with DT-related QTLs and introgressed intervals, will serve as useful resources for further functional dissection of the molecular mechanisms of drought stress response in rice.
\end{abstract}

Keywords: Drought tolerance, Introgression line, RNA sequencing, JA and GA pathway, Co-expression network, Rice

\footnotetext{
*Correspondence: zhouyongli@caas.cn; fubinying@caas.cn

${ }^{\dagger}$ Equal contributors

'Institute of Crop Sciences/National Key Facility for Crop Gene Resources and

Genetic Improvement, Chinese Academy of Agricultural Sciences, South

Zhong-Guan-Cun Street 12\#, Beijing 100081, China

Full list of author information is available at the end of the article
} 


\section{Background}

Rice (Oryza sativa L.) is a staple food for more than half of the world's population, especially in developing countries. Drought is the most serious environmental stress, limiting crop growth and productivity: drought-induced loss in crop yield probably exceeds losses from all other causes [1]. Drought tolerance (DT), therefore, is a major aim of rice breeding, especially in tropical Asian and African countries [2]. DT is a complex trait, and a number of quantitative trait loci (QTLs) for DT in rice have been identified [3]. However, breeding drought-tolerant rice is hard to achieve by conventional strategies, including marker-assisted selection. Understanding of the molecular mechanisms underlying DT is therefore needed for successful, knowledge-based crop improvement [4].

Determining the mechanisms directly involved in DT remains a challenging task because it involves several metabolic and morphologically adaptive pathways [5,6]. Abscisic acid (ABA) is an important phytohormone involved in drought stress tolerance in plants, whose mechanism in plant DT is relatively clear. Under drought stress, ABA-mediated stomatal closure is the mechanism used by plants to adapt to water deficiency [7-9]. Reactive oxygen species (ROS), including hydrogen peroxide, which are widely generated under stress, have been proposed to function as second messengers in ABA signaling in guard cells [10-13]. In guard cells, ABA-stimulated ROS accumulation activates plasma membrane calcium channels and triggers stomatal closure $[11,14]$. It was reported that jasmonic acid (JA) is also associated with stomatal closure under drought stress, the detailed molecular mechanisms remain elusive [15-17]. Though there is no evidence in relevance of gibberellic acid (GA) and stomatal closure under drought stress, reduction of GA levels and signaling has been shown to contribute to plant growth inhibition under several abiotic stresses, including cold, salt and osmotic stress $[18,19]$.

Many efforts have been made to identify the genes involved in drought stress tolerance in a number of plant species. Several drought-responsive genes encoding late embryogenesis abundant proteins, dehydration-responsive element binding protein, and protein phosphatase $2 \mathrm{C}$ were characterized as key components in the molecular network of DT [20]. In rice, genome-wide gene expression analyses identified many drought stress-responsive genes [21-24]. However, many of these drought stress-responsive genes have unknown functions, and details of their DT molecular mechanisms remain to be determined [20,25].

Rice is more sensitive to drought stress than other cereals, and large genotypic variation in DT exists within the cultivated rice gene pool and its wild relatives [2]. Selective introgression of DT donor segments into a drought-sensitive (DS) elite recurrent parent by backcrossing is an effective way to improve drought stress tolerance in rice $[2,26]$. In a previous study, we developed a DT introgression line (IL), H471, using the DT donor PSBRC28 (P28) and the DS recurrent parent Huang-Hua-Zhan (HHZ) (unpublished data). Compared with completely different genotypes with contrasting performance on the target trait, the ILs can largely reduce the genetic background noise in comparative transcriptomic analysis with the recurrent parent because the selected DT ILs carry a small number of genomic segments from a known DT donor [26]. Taking advantage of the combination of genome DNA re-sequencing and next-generation RNA sequencing (RNA-seq), ILs can effectively identify genes related to DT, thereby increasing our knowledge of the molecular mechanisms of this complex trait in rice [23]. In the present study, DT IL and its parental lines were used to analyze their transcriptome changes under drought stress comparatively, with the aim of extending our understanding of the genetic mechanisms of DT in rice.

\section{Results}

\section{Drought stress physiology of three rice genotypes}

At the tillering stage, there was no visible difference observed among H471, P28, and $\mathrm{HHZ}$ after 1 and 2 days of drought stress; however, obvious leaf rolling in $\mathrm{HHZ}$ was observed after 3 days of drought stress. This phenomenon was not observed in H471 and P28 until 4 days of drought stress (Figure 1A). The yield performance of three genotypes were remarkably reduced by drought stress compared with the well-watered control; however, H471 and P28 achieved $26 \%$ and $21 \%$ higher grain yields compared with $\mathrm{HHZ}$ under drought conditions (Figure 1B).

To investigate the physiological difference in DT of three genotypes, several indices of drought-induced effects on leaves at the tillering stage were measured. The water loss rate (WLR) from excised leaves was determined for the three genotypes: $\mathrm{H} 471$ and P28 showed relatively lower WLR than HHZ over a period of $10 \mathrm{~h}$ (Figure 2A). Accordingly, the relative water contents (RWCs) in H471 (76.9\%) and P28 (78.8\%) were significantly higher than that in $\mathrm{HHZ}(67.6 \%)$ after 3 days of drought stress (Figure 2B).

Stomatal closure is one of the first responses to drought conditions that might control plant dehydration [27]. To investigate the stomatal status of the three rice genotypes at the tillering stage under drought stress, leaf surfaces were examined using scanning electron microscopy (SEM). H471 and P28 had significantly higher stomatal closure rate than $\mathrm{HHZ}$ after 1 day of drought stress ( $p<0.01$, Kruskal-Wallis ANOVA, Dunn's multiple comparison test) (Figure $2 \mathrm{C}$ ). Relative electrolyte leakage (REL), which is an indicator of cell membrane injury, was also detected. The RELs of H471 and P28 were significantly lower than that of $\mathrm{HHZ}$ after 3 days of drought 

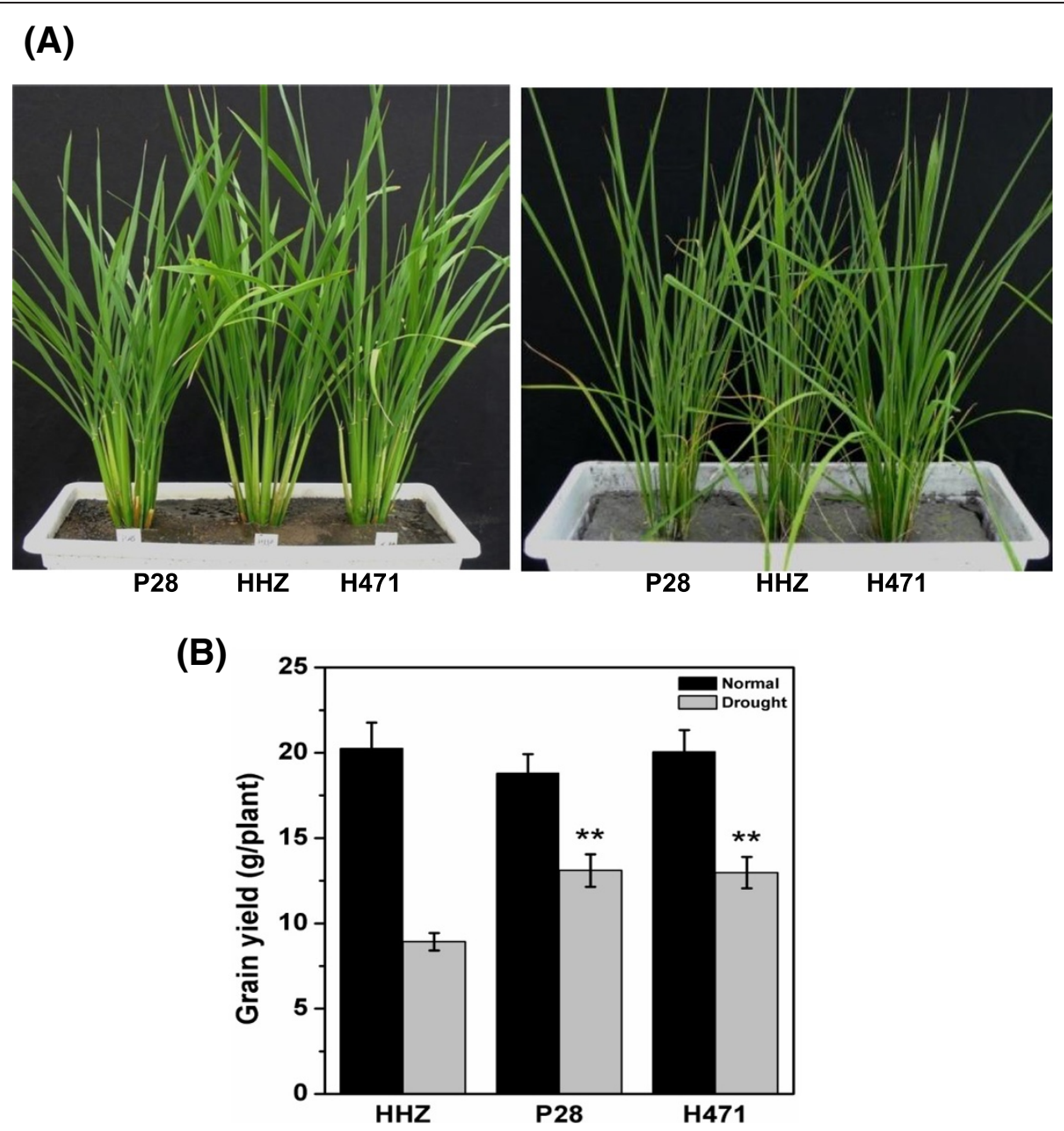

Figure 1 Phenotypes of three genotypes under well-watered and drought stress conditions. (A) Well-watered and 3 days drought stressed phenotypes of $\mathrm{P} 28, \mathrm{HHZ}$, and $\mathrm{H} 471$ at the tillering stage. (B) Grain yield performance of $\mathrm{HHZ}$, P28, and $\mathrm{H} 471$ under drought stressed and well-watered conditions. Each column represents mean \pm s.d. (nine replicates); ${ }^{* *} p<0.01$ versus HHZ (ANOVA, Dunnett's multiple comparison test).

stress (Figure 2D), indicating that $\mathrm{H} 471$ and P28 experienced significantly less cell membrane injury than HHZ under drought stress conditions. Additionally, the activities of catalase (CAT) and ascorbate peroxidase (APX) in H471 and P28 were significantly higher than that in HHZ under drought stress conditions (Figure $2 \mathrm{E}$ and $\mathrm{F}$ ), showing their active detoxification by reactive oxygen scavenging regulation in the two genotypes in response to drought [28]. Taken together, these results demonstrated that the DT of IL H471 was significantly improved over that of HHZ, reflecting the introgression of favorable alleles from DT donor P28 into the HHZ background.

\section{Global gene expression profiling of three genotypes} under well-watered and drought stress by transcriptome sequencing

At the tillering stage, total RNA from leaves of HHZ, P28, and $\mathrm{H} 471$ at 1 and 3 days of drought stress and its corresponding well-watered control were paired-end sequenced using Illumina sequencing technology. A total of 17.8-23.5 million reads of $100 \mathrm{bp}$ in length were generated for each sample, and the number of mapped reads were in the range of 14.4-19.6 million (Table 1). The unique matching ratio was in the range of $77.7-79.7 \%$ (Table 1), the unique matching reads were used for further analysis. The high-quality reads from individual libraries were mapped to the rice genome; more than 22,774 mapped genes per library were determined simultaneously.

The detected expressed genes in all samples were subjected to cluster analysis. As shown in Additional file 1, the three genotypes under 1 and 3 days of drought stress and control conditions were separated from each other: H471 and HHZ were clustered together in each condition subgroup, which was consistent with their similar genetic background. Based on this result, the transcriptomic response to $1 \mathrm{~d}$ and $3 \mathrm{~d}$ drought stress could be 


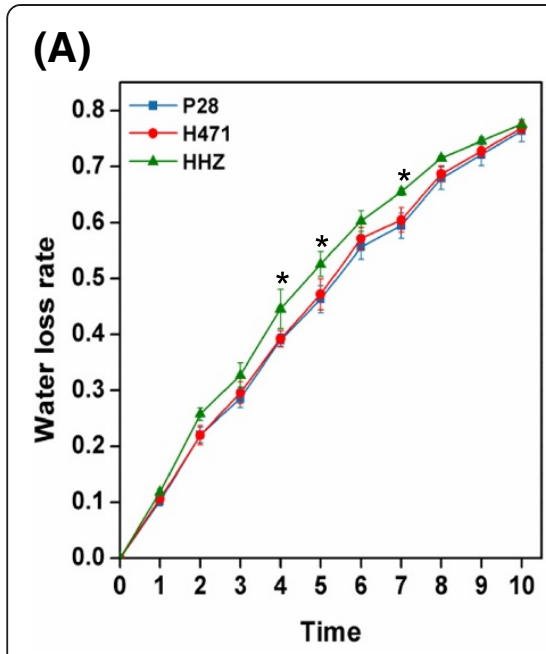

(D)

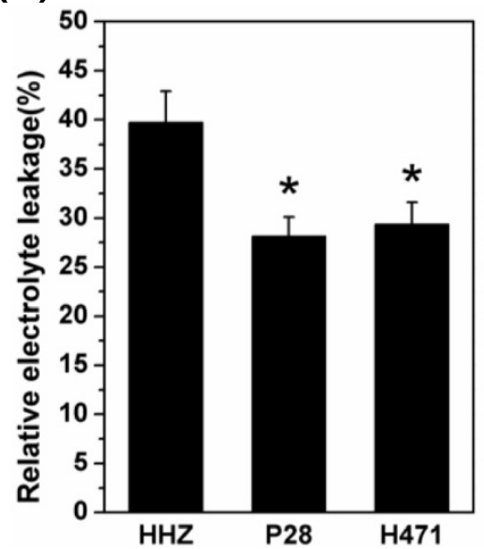

(B)

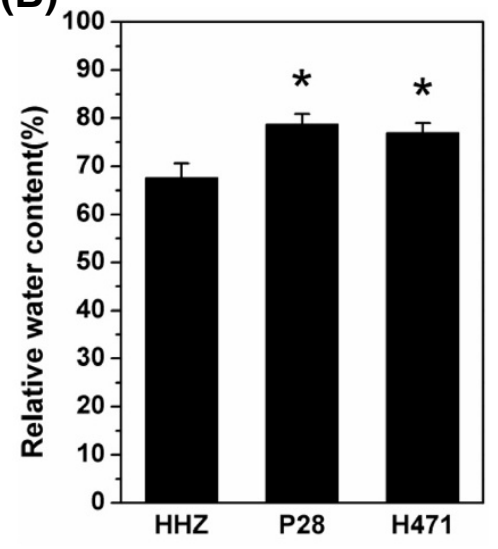

(E)

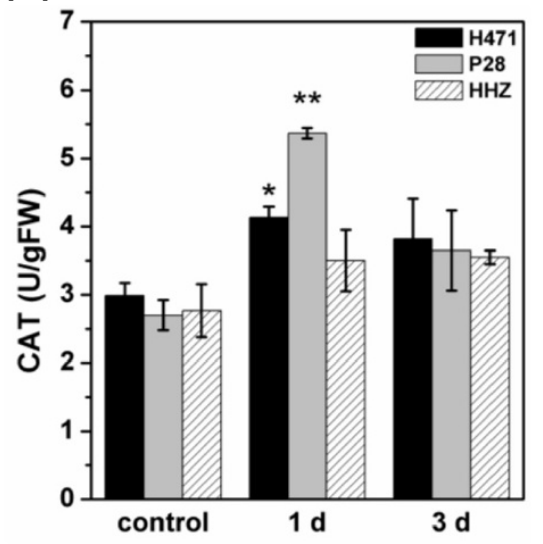

(C)

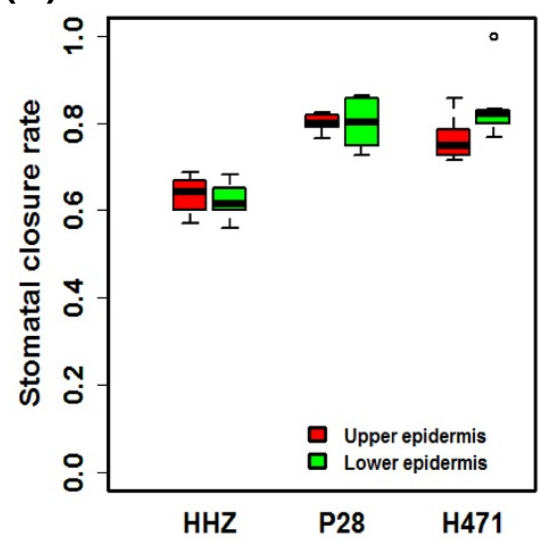

(F)

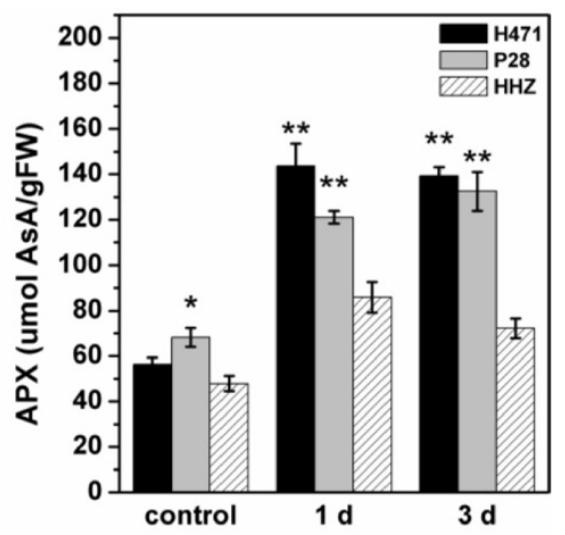

Figure 2 Differential drought physiological traits of H471, P28, and HHZ. (A) Water loss rate of P28, HHZ, and H471. For each replicate, 15 fully expanded leaves at the tillering stage were used in a triplicate experiment. (B) Relative water content of HHZ, P28, and H471 treated with 3 days of natural drought using the fully expanded leaves. (C) The stomatal closure rate in HHZ, P28, and H471 (upper epidermis: 6 randomly selected high power field for $\mathrm{HHZ}$; 5 for P28; 6 for H471; lower epidermis: 8 randomly selected high power field for HHZ; 6 for P28; 6 for H471). (D) Relative electrolyte leakage of HHZ, P28, and H471 after 3 days of drought stress. (E), (F) Activity of ROS-scavenging enzymes (CAT and APX) in three rice genotypes subjected to drought stress. Each column represents mean \pm s.d. (three replicates); ${ }^{* *} p<0.01$; ${ }^{*} p<0.05$ versus HHZ (ANOVA, Dunnett's multiple comparison test).

classified as mild stress response (MR) and severe stress response (SR), respectively, in consistent with the previous reports on abiotic stress gene profiling [29,30].

Correlation analysis was used to assess the quality of Illumina sequencing results between two replicates of each sample. The results indicated that the coefficient of correlation between the biological samples was high, supporting the reproducibility of the results (Additional file 2). To validate the Illumina sequencing results, quantitative real-time reverse transcription-PCR (qRT-PCR) was used to assess the expression levels for 27 genes of rice independently. The genes and primer sets used are shown in Additional file 3. RNA samples extracted from three additional replicate sets were used as templates. The high correlation $\left(R^{2}=0.93, p<0.01\right)$ between RNA-seq and qRT-RCR expression values indicated that there was a good agreement between both approaches (Additional files 4A and 4B).
Intrinsic transcriptome differences of H471 and P28 compared with $\mathrm{HHZ}$ prior to drought stress

Phenotypic differences resulting from gene expression variation have been observed in species [30,31]. To investigate the intrinsic differences in gene expression between the DT genotypes and the DS genotype, the gene expression levels in H471 and P28 were compared with that in HHZ under control conditions. Between P28 and HHZ, 1282 genes were identified as differentially expressed, which is consistent with their large genetic difference. However, there were only 343 genes detected to be differentially expressed between $\mathrm{H} 471$ and $\mathrm{HHZ}$ under normal growth conditions (Additional file 5). This is consistent with only a few of chromosome fragments being introgressed from P28 to HHZ. The genes with higher basal expression level in $\mathrm{H} 471$ compared with $\mathrm{HHZ}$ were mainly functionally enriched in oxidoreductase activity, lyase 


\begin{tabular}{|c|c|c|c|c|}
\hline Samples & Total filtered pair-end reads & Total mapped reads (\%) & Unique mapped reads (\%) & Total mapped genes \\
\hline HHZ-ck 1 & $2 \times 8,894,670$ & $14,403,823(81.4)$ & $13,766,465(77.8)$ & 23129 \\
\hline HHZ-ck 2 & $2 \times 9,326,860$ & $15,241,546(81.8)$ & $14,547,450(78.0)$ & \\
\hline P28-ck 1 & $2 \times 10,384,097$ & $16,978,445(81.9)$ & $16,233,203(78.3)$ & 23693 \\
\hline P28-ck 2 & $2 \times 10,326,754$ & $16,811,305(81.4)$ & $16,047,198(77.7)$ & \\
\hline H471-ck 1 & $2 \times 10,068,639$ & $16,551,528(82.2)$ & $15,823,734(78.6)$ & 23625 \\
\hline H471-ck 2 & $2 \times 10,083,264$ & $16,585,631(82.3)$ & $15,852,617(78.6)$ & \\
\hline $\mathrm{HHZ}-1 \mathrm{~d} 1$ & $2 \times 11,727,199$ & $19,581,596(83.5)$ & $18,660,210(79.6)$ & 23078 \\
\hline $\mathrm{HHZ}-1 \mathrm{~d} 2$ & $2 \times 9,958,934$ & $16,628,254(83.5)$ & $15,864,006(79.7)$ & \\
\hline P28-1d 1 & $2 \times 11,594,160$ & $19,305,500(83.3)$ & $18,463,197(79.7)$ & 22774 \\
\hline P28-1d 2 & $2 \times 10,484,144$ & $17,435,333(83.2)$ & $16,669,090(79.6)$ & \\
\hline H471-1d 1 & $2 \times 9,853,652$ & $16,126,140(81.9)$ & $15,390,608(78.1)$ & 23320 \\
\hline $\mathrm{H} 471-1 \mathrm{~d} 2$ & $2 \times 10,868,092$ & $17,847,671(82.2)$ & $17,055,619(78.5)$ & \\
\hline $\mathrm{HHZ}-3 \mathrm{~d} 1$ & $2 \times 10,071,423$ & $16,584,356(82.4)$ & $15,857,639(78.8)$ & 22976 \\
\hline $\mathrm{HHZ}-3 \mathrm{~d} 2$ & $2 \times 10,042,756$ & $16,586,139(82.6)$ & $15,844,907(78.9)$ & \\
\hline P28-3d 1 & $2 \times 10,271,850$ & $16,943,591(82.5)$ & $16,175,470(78.8)$ & 23017 \\
\hline P28-3d 2 & $2 \times 10,093,202$ & $16,568,958(82.1)$ & $15,817,307(78.4)$ & \\
\hline H471-3d 1 & $2 \times 10,087,890$ & $16,669,962(82.7)$ & $15,912,430(78.9)$ & 22974 \\
\hline $\mathrm{H} 471-3 \mathrm{~d} 2$ & $2 \times 9,948,863$ & $16,440,601(82.7)$ & $15,735,994(79.1)$ & \\
\hline
\end{tabular}

Note: ck indicates well watered control; $1 \mathrm{~d}$ and $3 \mathrm{~d}$ indicate the drought treatment time; 1 and 2 in the first row indicate two replicates of each sample.

activity, carboxylic acid metabolic process, response to stress, and cofactor binding (Additional file 6-1).

These constitutively differentially expressed genes in P28 and H471 compared with HHZ under control conditions could be classified into two groups based on their expression patterns under drought stress conditions. The first group comprised those that were unresponsive to drought stress, including 503 and 74 genes from P28 and H471, respectively, which are functionally enriched in redox regulation and apoptosis-associated proteins (Additional file 7-1 and 7-2). The second group comprised 779 and 269 genes differentially expressed in P28 and H471 after at least 1 or 3 days of drought stress (Additional file 7-3 and 7-4). Among them, 67 genes (Additional file 7-5) with higher basal expression in both P28 and H471 were functionally enriched in oxidoreductase and lyase activity. Further analysis indicated that 10 of these genes were colocalized in the introgressed regions, implying their positive role in the response to drought stress.

\section{Comparative transcriptome profiling of three genotypes under drought stress}

To determine the similarities and differences in droughtinduced transcriptomes in the three genotypes, the gene expression alterations in the three genotypes under 1 and 3 days of drought stress compared with their respective controls were analyzed. In H471, P28, and HHZ, 7862,
7717, and 7625 DEGs were identified, respectively, after 1 or 3 days of drought stress.

After 1 day of drought stress, there were 5617, 5849, and 5579 DEGs detected in H471, P28, and HHZ, respectively. Venn diagram analysis indicated that 3945 DEGs (1966 up-regulated and 1979 down-regulated) were commonly regulated in the three genotypes by 1 day of drought stress (Table 2, Figure 3A and B) which accounted for $70.23 \%, 67.45 \%$ and $70.71 \%$ of total DEGs in H471, P28 and HHZ, respectively, indicating that the mild drought stress response of the different genotypes is mostly the same. GO analysis showed that the commonly up-regulated genes were functionally enriched in the regulation of the biological process, signaling process, and carbohydrate metabolic process (Additional file 6-2); while the shared down-regulated genes in the three genotypes were mainly associated with cellular protein metabolic process, phosphate metabolic process, and transport (Additional file 6-3).

After 3 days of drought stress, 5290, 4957, and 5116 genes were identified as DEGs in H471, P28, and HHZ, respectively (Table 2), showing relatively fewer DEGs could be detected at the SR phase compared with the MR phase. Among them, 3308 DEGs (1484 up-regulated and 1824 down-regulated) were commonly regulated in three genotypes at the SR phase which accounted for $62.53 \%, 66.73 \%$ and $64.66 \%$ of total DEGs in H471, P28 and $\mathrm{HHZ}$, respectively (Figure $3 \mathrm{C}$ and D). GO analysis 
Table 2 Summary of differentially expressed genes in H471, HHZ and P28 under 1d and 3d drought stress compared with its respective well-watered control

\begin{tabular}{cccccccc}
\hline & \multicolumn{1}{c}{ 1d } & & & \multicolumn{2}{c}{ 3d } \\
\cline { 2 - 4 } & Up-regulated & Down-regulated & Sub-total & & Up-regulated & Down-regulated & Sub-total \\
\hline H471 & 2785 & 2832 & 5617 & & 2486 & 2804 & 5290 \\
P28 & 2779 & 3070 & 5849 & & 2122 & 2835 & 4957 \\
HHZ & 2702 & 2877 & 5579 & & 2403 & 2713 & 5116 \\
Common & 1966 & 1979 & 3945 & & 1484 & 1824 & 3308 \\
\hline
\end{tabular}

of these DEGs indicated that carbohydrate metabolic process, regulation of cellular process, transcription, macromolecule modification, cellular protein metabolic process, and transport were highly enriched in the SR DEGs (Additional files 6-4 and 6-5). Taken together, the results showed that broad functional categories of genes were commonly involved in drought stress response.

Effect of introgression on the transcriptome of $\mathrm{H} 471$ in response to drought stress

The backcross introgression strategy is widely used for crop improvement. The introgressions combine the genetic background of the recurrent parent, which could result in novel changes in gene expression. To evaluate the effect of introgression on transcriptome of H471 under drought stress, the genome-wide gene expressions in H471 and HHZ under drought stress were compared. The results indicated that 460 and 380 genes were upand down-regulated, respectively, in H471 compared with HHZ under drought stress (Additional file 8). Only a small proportion of the DEGs (128 up-regulated, 103 down-regulated) colocalized with the introgressed regions (Additional file 8), indicating introgression could contribute to new DT expression phenotypes in $\mathrm{H} 471$ relative to $\mathrm{HHZ}$.

\section{(A)}

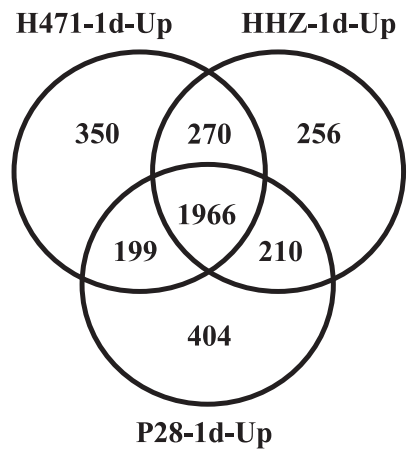

(C)

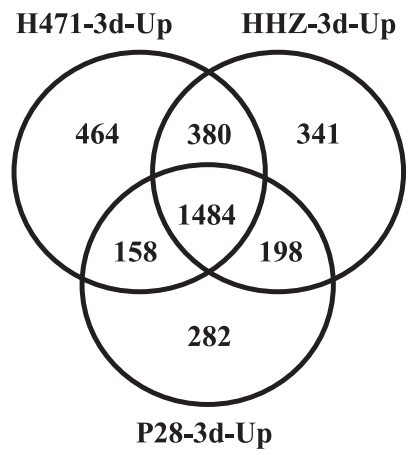

(B)

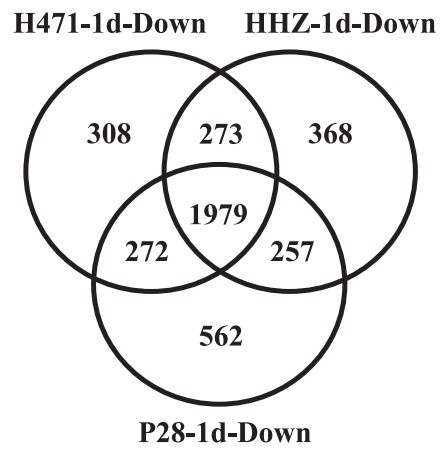

(D)

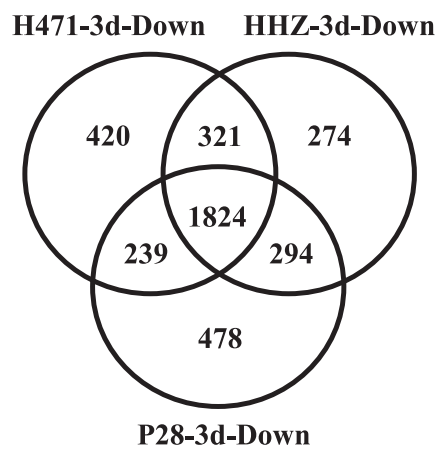

Figure 3 Venn diagram of differentially expressed genes (DEGs) in H471, HHZ, and P28 under 1 and 3 days of drought stress. DEGS were identified with adjusted $p$-value $<0.001$. (A), (B), (C), and (D) show the Venn diagram results for the three genotypes of 1 day up-regulated, 1 day down-regulated, 3 days up-regulated, and 3 days down-regulated genes, respectively, under drought stress. 
GO enrichment analysis of these DEGs highlighted the following functional categories: signaling transduction, transcription regulation, stress response, hormones (GA, JA) signal transduction, and ROS homeostasis (Additional file 9).

Genes related to signaling transduction: 18 genes encoding receptor kinases (RKs) were differentially expressed in H471 vs. HHZ under drought conditions. Among them, two genes encoding cysteine-rich receptor-like protein kinases (RLKs) (LOC_Os07g43560, LOC_Os07g43570) were up-regulated in $\mathrm{H} 471$ vs. $\mathrm{HHZ}$ and one was repressed (LOC_Os04g30040). Eight genes encoding leucine-rich repeat transmembrane protein kinases were up-regulated and five were down-regulated in $\mathrm{H} 471$ relative to $\mathrm{HHZ}$. Additionally, 26 genes encoding kinase proteins were differentially expressed in H471 vs. HHZ under drought stress. These included four genes encoding calmodulin-dependent protein kinases (CDPKs) (LOC_Os03g20380, LOC_ Os03g43440, LOC_Os05g26870, and LOC_Os10g39420). Three genes encoding EF hand calcium-binding proteins (OsCML15, OsCML18, and OsCML31) were up-regulated in $\mathrm{H} 471$, and two genes encoding $\mathrm{CaLB}$ domain proteins (LOC_Os08g20544, LOC_Os02g27130) were down-regulated in $\mathrm{H} 471$ compared with $\mathrm{HHZ}$ under drought stress, indicating their different roles in the drought stress response.

Phytohormone related proteins: 16 genes encoding ten JA and six GA related proteins were differentially expressed in H471 vs. HHZ under drought stress (Additional file 9). Kyoto encyclopedia of genes and genomes (KEGG) analysis of these DEGs showed that alpha-linolenic acid metabolism (ko00592), which is associated with JA synthesis, was highlighted in H471 (Additional file 10). Eight JA biosynthesis-related genes, encoding four lipoxygenase (LOX), one alpha-dioxygenase (DOX1), one acyl-CoA oxidase (ACX), one enoyl-CoA hydratase/3-hydroxyacyl-CoA dehydrogenase (MFP2) and one acetyl-CoA acyltransferase (fadA), were differentially expressed in H471 compared with HHZ under drought stress. Meanwhile, two genes (OsJAZ1 and OsJAZ7) involved in the JA signaling pathway were also significantly up-regulated in H471 vs. $\mathrm{HHZ}$ under drought stress. Consistently, the JA content in H471 was significantly higher than that in $\mathrm{HHZ}$ under drought stress, even though the JA contents in all three genotypes were repressed by drought compared with their respective control (Figure 4, Additional file 11). A GA biosynthesis-related gene, encoding putative gibberellin 20-oxidase 2 (GA20ox2) was significantly up-regulated after 1 day of drought stress, while a GA deactivationrelated gene encoding gibberellin 2-oxidase was evidently up-regulated after 3 days of drought stress in all genotypes. Importantly, four putative gibberellin receptor encoded genes were detected to be differentially expressed in H471 compared with HHZ under drought stress. These results indicated that the JA and GA pathway is involved in drought stress tolerance in the DT IL H471.

Transcription regulation related genes: 36 transcription factor (TF) genes were differentially expressed between $\mathrm{H} 471$ and $\mathrm{HHZ}$ under drought stress. Among them, three AP2/EREBP genes (LOC_Os01g64790, LOC_Os03g08470, LOC_Os04g57340) were significantly up-regulated in H471, which were previously identified to be highly involved in drought and salt stress [32]; OsWRKY4 (LOC_Os01g53040) and OsWRKY9 (LOC_Os01g18584) were highly expressed in $\mathrm{H} 471$ relative to $\mathrm{HHZ}$ under drought; these two genes were associated with biotic stress tolerance in a previous study [33,34]. The others included four MYB TFs, five bHLH TFs, and seven NAC proteins, most of which were up-regulated in H471 compared with HHZ under drought, implying their positive role in the drought stress response.

Genes encoding redox regulation-related proteins: A set of 19 genes related to redox regulation were enriched in H471 compared with HHZ under drought stress. These genes included those encoding ascorbate peroxidase, oxidoreductase, peroxidase precursor, glutathione S-transferase, and glutathione synthetase (Additional file 9), showing that redox regulation is involved in the molecular mechanisms of drought stress tolerance.

Genes related to carbohydrate metabolism and osmotic adjustment: 9 genes encoding UDP-glucoronosyl and UDP-glucosyl transferase (UGT) family proteins and genes encoding three osmotins and one malate synthase were differentially expressed in $\mathrm{H} 471$ vs. HHZ under drought. These genes were previously reported to be involved in sugar metabolism and detoxification $[35,36]$ and osmotic adjustment [37].

\section{Co-regulatory gene networks of $\mathrm{H} 471$ in response to drought stress}

To explore the genetic networks associated with DT, all DEGs belonged to the above function categories in H471 compared with $\mathrm{HHZ}$ under drought stress (Additional file 9) were subjected to co-expression network analysis. Forty of them were found to be co-regulated and formed a complex network (Figure 5). In this network, the genes could be separated into four groups according to their putative functions. Group A was enriched in stress signaling transduction, including 13 genes encoding receptor kinases, protein kinases, and $\mathrm{Ca}^{2+}$ related protein. Four genes in group B were functionally involved in hormone signaling pathways: three genes encoding lipoxygenases (LOXs) and one gene encoding GA20ox2; LOX is involved in JA biosynthesis and signaling [38,39]; and GA20ox2 is related to the biosynthesis of gibberellin [40]. The genes in group $\mathrm{C}$ were enriched in transcription regulation, including two NAC, one MYB, three CCT/B-box zinc finger proteins, and one HLH type TFs. The group D genes were 


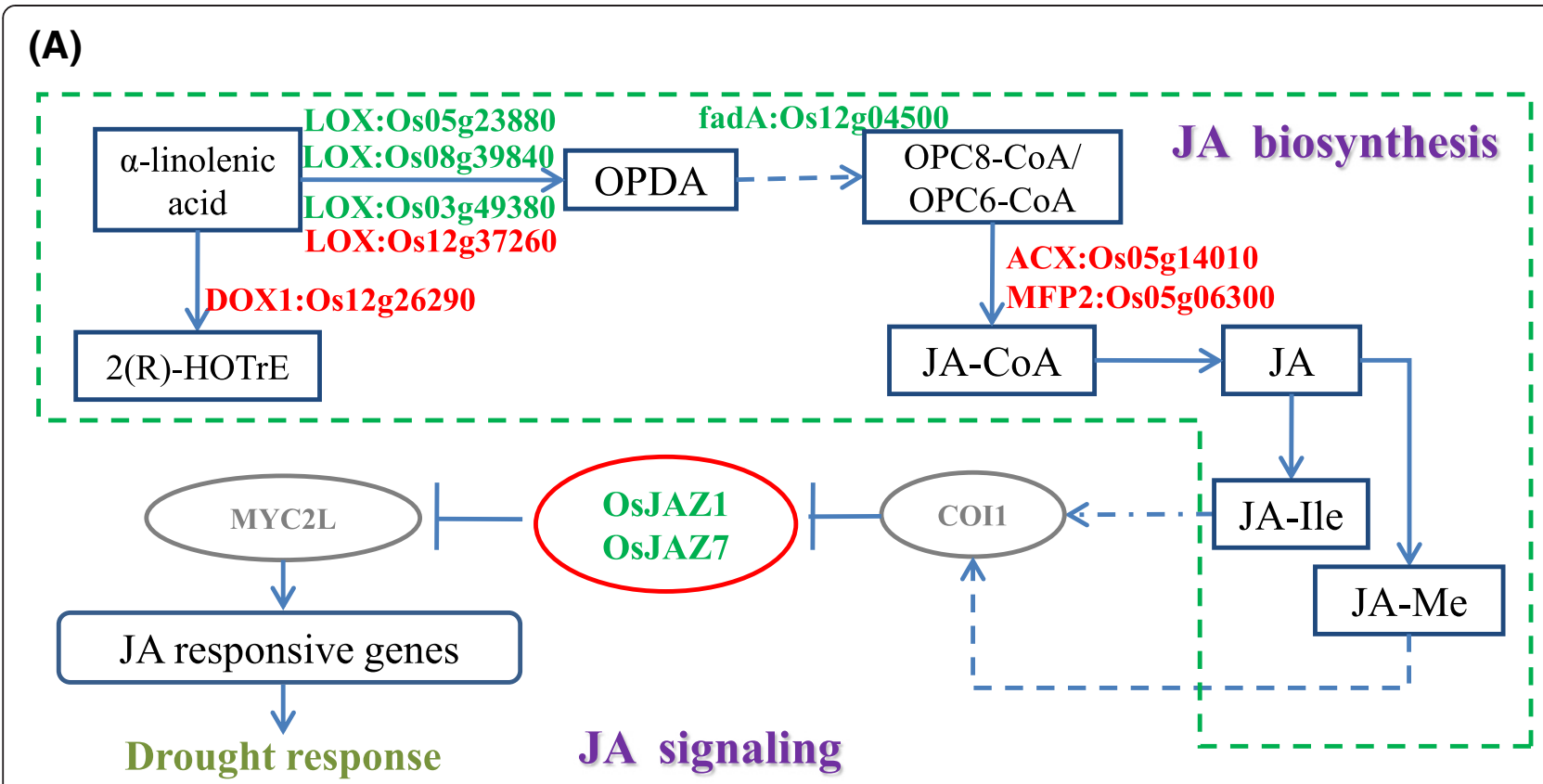

(B)

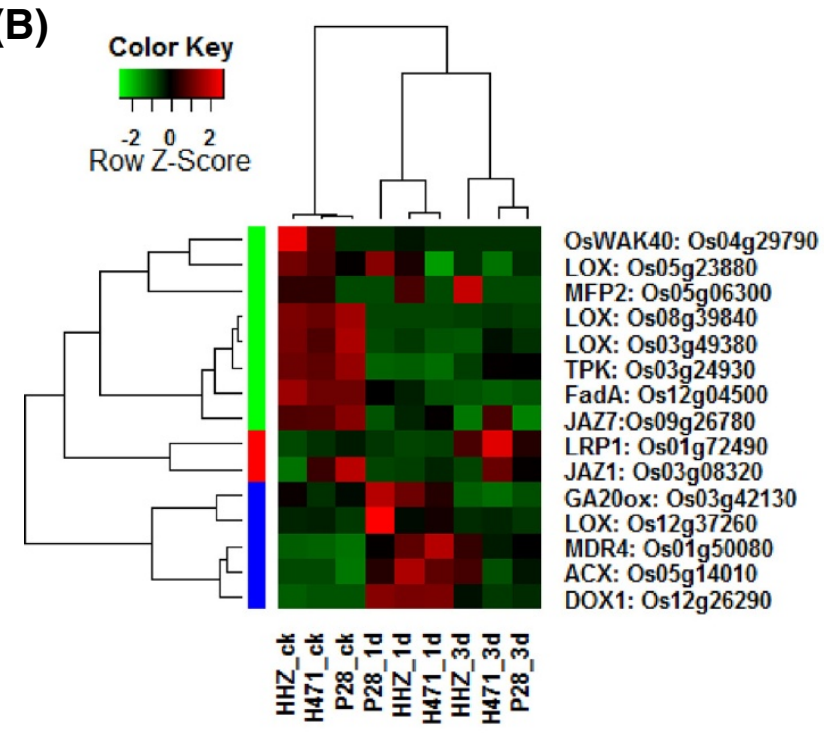

(C)

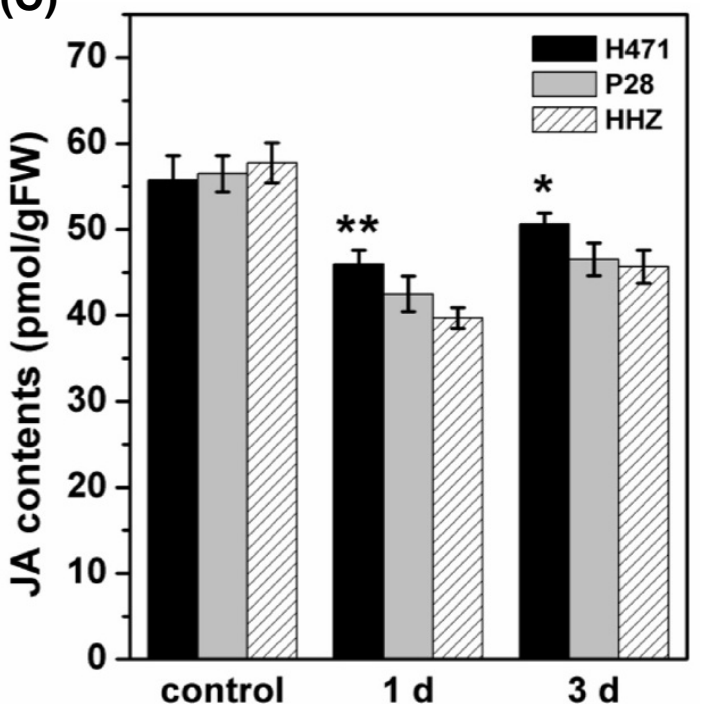

Figure 4 Jasmonic acid (JA) related pathways may play key roles in drought tolerance in H471. (A) JA synthesis and signaling pathways that contain differentially expression patterns between $\mathrm{H} 471$ and $\mathrm{HHZ}$ under drought conditions. Gene names in red and green color indicate up- and down-regulated in response to drought stress, respectively. (B) Hierarchical clustering of JA-related genes and some co-regulated genes. The color scale indicates the expression value. (C) JA contents in $\mathrm{H} 471, \mathrm{HHZ}$, and P28 under drought conditions. Each column represents mean \pm s.d. (three replicates); ${ }^{* *} p<0.01 ;{ }^{*} p<0.05$ versus HHZ (ANOVA, Dunnett's multiple comparison test).

mainly involved in ion transport, including two high affinity $\mathrm{K}^{+}$transporter $5 \mathrm{~s}$, three $\mathrm{ABC}$ transporters, and an ATP synthase subunit $C$ family protein; and redox homeostasis, including peroxidase superfamily proteins, two glutathione S-transferases, and one short-chain dehydrogenase/reductase; and carbohydrate metabolism, including UDP-glycosyltransferase and malate synthase. These coregulated genes in group $\mathrm{D}$ were evidently downstream in the drought stress tolerance mechanism of H471.
Colocalization of DT related DEGs in the introgression fragments and QTL intervals

According to the results of genotyping by resequencing, 26 P28 fragments were introgressed into H471 (Figure 6). We analyzed the previously reported DT-related QTLs based on the Gramene QTL database (http://archive.gramene. org/qtl/) compared with the introgression intervals in H471. The results indicated that 39 known DT QTLs were co-localized in the introgressed regions in H471 (Figure 6). 


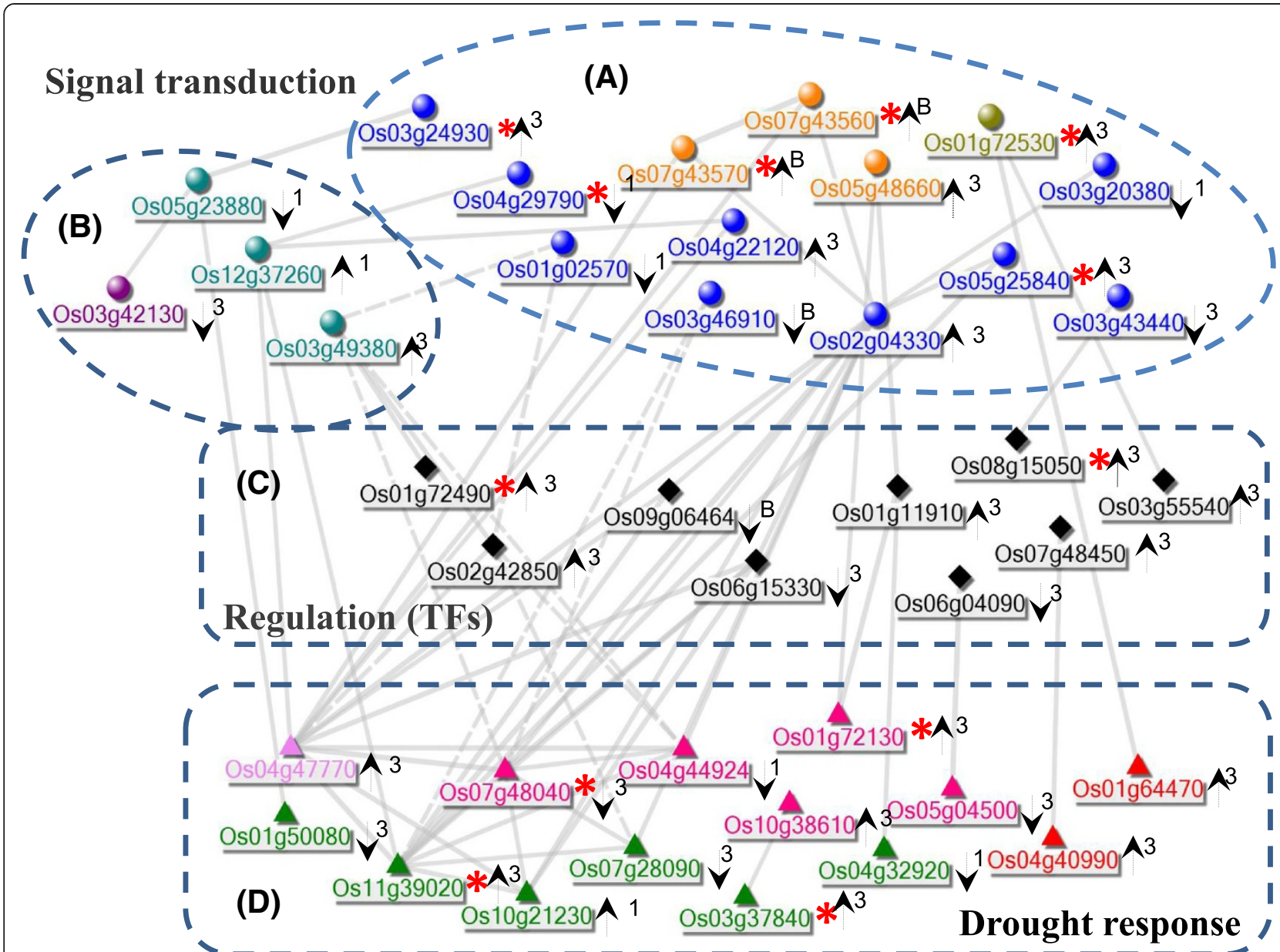

Figure 5 Co-regulatory networks of genes differentially modulated in $\mathrm{H} 471$ under drought stress (false discovery rate 0.05$)$ ). Co-regulation analysis was based on the calculation of pairwise Pearson correlation coefficient (PCC) of logarithmic expression values (with a cutoff of 0.75 ) in Rice Oligonucleotide Array Database. Four subgroups, indicated with letters from A-D which also distinguished by shapes, were identified as their putative functions. Different colors indicate genes with unique function class: olive, Ca2+ signal related; gold, receptor kinase; blue, kinase; violet, TF; green, $\mathrm{K}+$ transporter; Pink, redox regulation; black, osmotin; red, carbohydrate metabolism. The thickness of the edges is proportional to the PCC. Asterisks indicate the genes located in the introgression segment from P28. Arrows indicate up- and down-regulated in response to drought stress, respectively; and 1, 3 and $B$ with arrows show 1 day, 3 days, both 1 and 3 days drought stress, respectively.

Correspondingly, all DEGs, including those differentially expressed in H471 compared with HHZ under control, 1 day or 3 days of drought stress conditions, were mapped onto the rice chromosome: 289 of these DEGs were localized on the introgressed intervals. Furthermore, 205 DEGs were localized onto 15 DT-related QTL intervals that overlap with the introgressed fragments in H471 (Additional file 12). Seven genes on the introgressed region of chromosome 1 were detected to be significantly up-regulated in $\mathrm{H} 471$ relative to HHZ after 3 days of drought stress: bHLH TF, lateral root primordium (LRP) protein, beta-1,3-glucanase 3, glycosyl hydrolase, glutathione S-transferase, and bidirectional amino acid transporter. Several drought-related QTLs, including those for leaf rolling, osmotic adjustment, and relative water content [41-43] were colocalized on this region; thus, up-regulation of these seven genes in $\mathrm{H} 471$ could be positively related to physiological and metabolic adaptation to drought stress. There was also a cluster of 10 genes located on the introgressed segment of chromosome 4 that had no corresponding DT QTLs. These genes included eight wall-associated kinase (WAK) family proteins and two cysteine-rich RLKs were found to be constitutively down-regulated in H471 compared with HHZ. It was evidenced that WAKs and cysteine-rich RLKs are essential for the normal regulation of cell enlargement and abiotic stress sensing [44,45], reduced expression of these genes in H471 might negatively affect cell growth and the stress response.

\section{Discussion}

The DT IL showed enhanced drought stress tolerance ILs have been widely used in genetic analysis and molecular breeding $[26,46]$. Drought-selected ILs have 


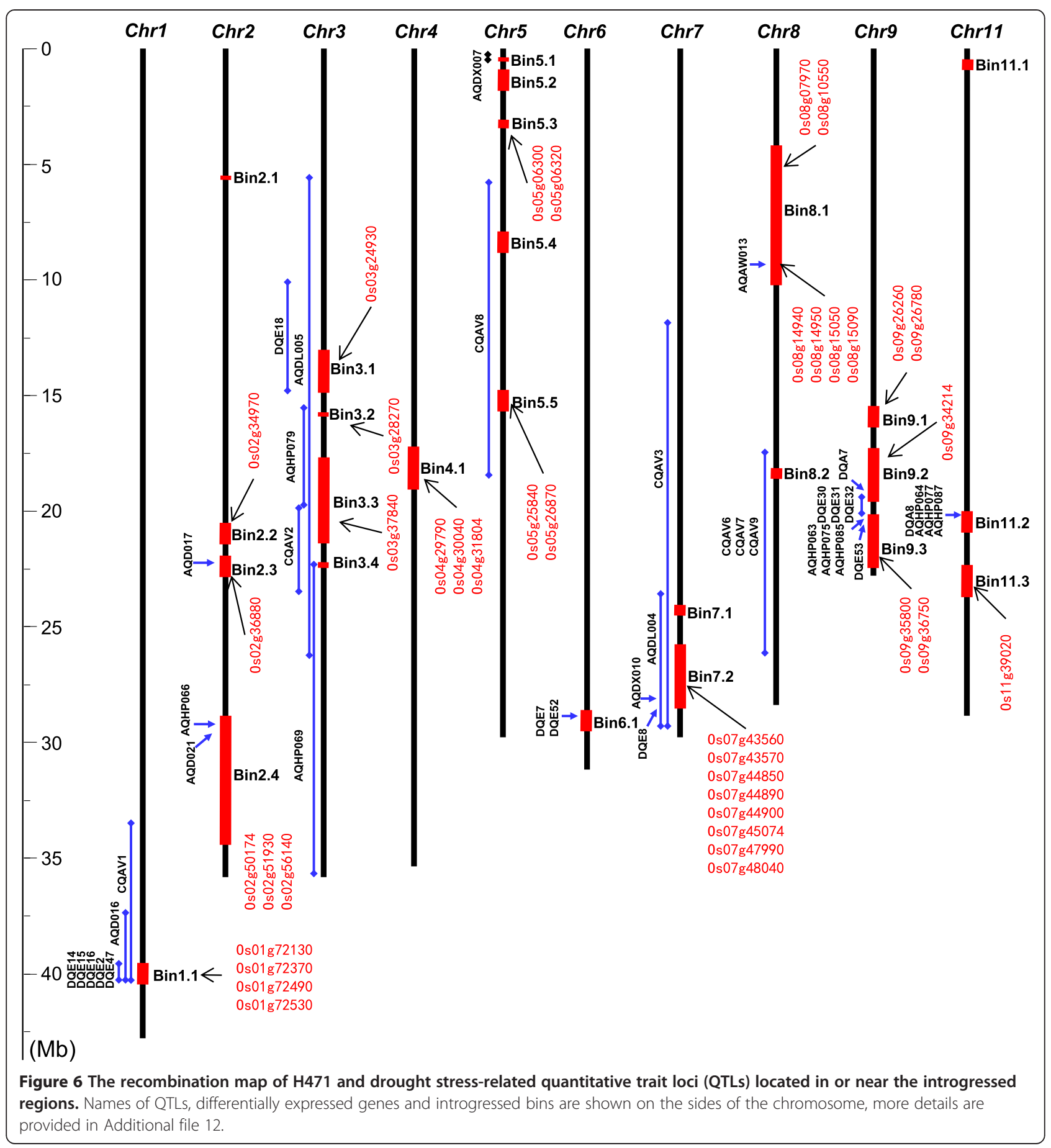

provided a useful genetic resource to improve our understanding of the genetic and molecular basis of DT in rice [2]. In the present study, the DT IL H471 and donor P28 were more tolerant to drought stress than the recurrent parent HHZ. Under drought stress, H471 and P28 showed delayed leaf rolling, lower RWL, higher RWC, higher stomatal closure rate, relatively lower cell membrane injury, and with significantly higher CAT and APX activities than HHZ, indicating that $\mathrm{H} 471$ and P28 underwent complex morphological and physiological changes to resist drought stress [47]. These results showed that $\mathrm{H} 471$ and P28 used a typical DT strategy to cope with drought stress by limiting water loss and the enhanced DT of H471 over HHZ was caused by introgression of favorable alleles from donor P28. 
Genome-wide transcriptome reprogramming of $\mathrm{H} 471$ and its parental lines in response to time-series drought stress A comparative transcriptome analysis of three genotypes under 1- and 3-day drought stress indicated that the DT and DS genotypes shared a large proportion of drought-induced DEGs, revealing common drought stress-responsive processes. GO analyses showed that commonly up-regulated genes at the MR phase were highly enriched in signaling transduction and transcription regulation, consistent with previous reports that abiotic stress triggered the MR phase of transcriptome alterations of genes related to signaling cascades, including receptor kinases, transcription factors and components of calcium signaling $[30,48]$. However, after 3 days of drought stress, the common DEGs were mainly involved in carbohydrate metabolic process, posttranslational protein modifications, transport, and redox regulation, implying that downstream genes related to metabolite adaptation, ROS homeostasis, and posttranscriptional regulation were involved in the severe drought stress response, in accordance with previous results $[48,49]$.

\section{Effect of introgression on the transcriptome in response to drought stress}

The DEGs on the introgressed regions in $\mathrm{H} 471$ relative to its recurrent parent $\mathrm{HHZ}$ could have direct effects on its DT phenotype changes. Identification of those DEGs colocalized with identified DT-related QTLs provides useful data for DT molecular breeding and gene functional dissection. Meanwhile, it was reported that donor introgressions combine recurrent parental alleles that might result in novel changes in expression, and many genes showing non-parental expression patterns were identified outside of the introgressed fragments [50]. In this study, a set of genes in the DT introgression line $\mathrm{H} 471$ were exclusively differentially expressed compared with its parental lines, especially under drought stress, showing a unique expression related to the DT phenotype. These expression changes beyond the introgression regions might correspond to the well-known transgressive or nonparental expression in hybrid crop plants [51]. However, it was determined that the genome-wide expression changes in an IL might result from activation of transposon mPing [52] or transgressive siRNA [53]. The molecular mechanisms of this non-parental expression alteration, especially under drought stress, need to be further elucidated.

\section{A complex genetic network including the JA and GA pathway is involved in drought stress tolerance}

Drought stress tolerance is a complex trait and involves many genes. Hence, deciphering the molecular mechanisms underlying DT in plants is a challenging task. Genome-wide identification of drought-responsive regulons in contrasting DT genotypes with similar genetic backgrounds could help to dissect novel genetic components involved in DT. We comparatively analyzed the differential gene expression between $\mathrm{H} 471$ and $\mathrm{HHZ}$ under drought stress: 840 genes were differentially expressed, indicating substantial transcriptome reprogramming in H471 under drought stress compared with HHZ.

A set of genes related to signaling transduction including RKs, CDPKs, and CBPs were up-regulated in H471 compared with HHZ. RLKs play an important role in plant growth and responses to abiotic stresses by activating initial signaling transduction [45]; CDPKs and CBPs are the main components in the stress signaling pathway, which acts by modulating ABA signaling and reducing the accumulation of ROS [54,55]. Up-regulation of these genes in $\mathrm{H} 471$ implied their positive role in DT by enhancing signaling pathways under drought stress.

Plant hormones, including ABA, GA and JA, play key roles in their ability to adapt to changing environments $[15,18,19,56]$. GO and KEGG analyses revealed that genes related to JA biosynthesis and the signaling pathway were differentially expressed in $\mathrm{H} 471$ relative to HHZ under drought stress, implying that JA was involved in drought response. JA plays an important role in plant growth, development and stress response [57]. The JA signaling and biosynthesis genes were found to be significantly regulated by drought in Arabidopsis [58] and rice [59]. In this study, the JA levels in all three genotypes were repressed by drought compared with their control, this result indicated that JA is probably not required at high concentration under drought stress, and an increase in JA content might negatively affect plant growth as suggested by previous reports $[58,60]$. However, the JA content was significantly higher in H471 than in HHZ and P28 under drought stress, which correlated with the increased expression of JA biosynthesis genes in H471 relative to $\mathrm{HHZ}$, demonstrating that JA plays an important role in DT. Previous studies indicated that JA affected the transcript levels of genes related to antioxidants under water stress [61]; relative higher JA content in H471 might enhance drought tolerance by modulating antioxidant homeostasis.

It was shown that JA could interact synergistically and antagonistically with other phytohormones [60,62]. In this study, differentially expressed GA-related genes in H471 compared with $\mathrm{HHZ}$ under drought stress provide evidence for the role of GA metabolism and the regulation of the GA signaling pathway on exposure to drought stress which consistent with latest study. Emerging evidence for interaction of the GA-signaling molecule DELLA with components of the signaling pathway for the stress hormone JA [19] suggests that GA signaling might integrate multiple hormone signaling pathways in response to drought stress, however the crossroads of these interactions still remain to be elucidated. 
TFs are hub regulators in response to biotic and abiotic stresses. Several TFs, including AP2/EREBPs, WRKYs, bHLHs, and NACs, were highly up-regulated in H471 compared with $\mathrm{HHZ}$ under drought stress. All these TFs were previously reported to be involved in transcription regulation of abiotic stresses tolerance [20,63]. Importantly, AP2/EREBPs, WRKYs, and NACs are key regulators of ABA-mediated stomatal closure and, hence, drought responses $[64,65]$. bHLHs play an important role in the JA-mediated regulatory network of the abiotic stress response [66]. Differential expression of these TFs in $\mathrm{H} 471$ relative to $\mathrm{HHZ}$ implied that both ABA and JA play a central role in regulating drought stress tolerance in $\mathrm{H} 471$.

Co-expression analysis showed that there were core DT genes forming a complex network in H471 (Figure 5), including genes related to signaling transduction, the JA pathway, and TFs, and those involved in downstream functions, such as carbohydrate metabolism, ion transport, and ROS regulation. In the network, a bHLH TF (LOC_Os01g11910) is connected to a receptor-like kinase (LOC_Os05g48660) and a hub 5-AMP-activated protein kinase (LOC_Os02g04330), interacts with glutathione S-transferase (LOC_Os01g72130), LZ-NBS-LRR protein (LOC_Os11g39020), and peroxidase (LOC_Os07g48040), and is then further connected with two JA biosynthesis lipoxygenases (LOC_Os03g49380 and LOC_Os12g37260). All these genes form a complex interacting network. However, their real interactions need to be confirmed by further experiments, including yeast-two-hybridization screening. These co-expressed gene networks provide useful information for dissecting the molecular mechanisms underlying drought stress tolerance in rice.

\section{Conclusions}

In this study, a DT IL and its parental lines, including the DT donor and DS recurrent parent, were used to characterize the differences of leaf transcriptome dynamics under 1 and 3 days of drought stress at the tillering stage using high-throughput RNA sequencing. Drought induced transcriptome reprogramming in a DT IL H471 could result from introgressed chromosome segments from the DT donor P28, and the differentially expressed genes in the $\mathrm{H} 471$ relative to the HHZ under drought stress might contribute to the enhanced drought tolerance, finally improved yield performance under stress. Co-expression analysis revealed a complex regulatory network, including genes related to the signaling transduction, JA and GA pathways, transcription regulation, redox control and osmotic adjustment, involved in drought stress tolerance. The data obtained in this study could extend our understanding of the molecular mechanisms of DT in rice.

\section{Methods}

\section{Plant materials and experimental treatments}

Three rice genotypes were used in this study. HHZ is a widely used indica inbred rice in South China, with high yield and good quality, which is sensitive to drought stress. P28 is an indica rice variety from the Philippines and is well adapted to drought stress. $\mathrm{H} 471$ is a $\mathrm{BC}_{1} \mathrm{~F}_{5}$ droughttolerant IL with a few chromosomal fragments introgressed from the donor parent P28 into background of the recurrent parent HHZ. Genome-wide single nucleotide polymorphism analysis by re-sequencing showed that $\mathrm{H} 471$ differs from $\mathrm{HHZ}$ at 26 genomic segments from $\mathrm{P} 28$, with sizes in the range of $57-6057 \mathrm{~kb}$ (Figure 6). The recombination bins were judged as described in Huang et al. [67], using the genome-resequencing data of $\mathrm{HHZ}$, P28, and H471 (unpublished data).

To evaluate the DT performance of the three genotypes, a pot experiment was arranged in a randomized complete block design with three treatments (well-watered, 1-day drought stressed, and 3-day drought stressed) and six replications or pots in a greenhouse at the Institute of Crop Sciences of Chinese Academy of Agricultural Sciences (Beijing, China). The days were counted after the available water content (AWC) in the soil reached 20\% to allow drought measurements at precisely determined intervals. Three healthy seedlings of the three genotypes were transplanted equidistantly into a strip pot $(15 \mathrm{~cm}$ in height and $50 \mathrm{~cm}$ in diameter) filled with $2 \mathrm{~kg}$ of sterilized field soil, which contained about $50 \%$ AWC of the soil, as measured by soil moisture meters (TZS-W, Zhejiang Top Instrument Co. Ltd). Seedlings of each genotype were planted in six pots giving a total of six plants; all plants were grown with $14 \mathrm{~h}$ daylight at $28^{\circ} \mathrm{C}$ and a 10 -h dark period at $25^{\circ} \mathrm{C}$ under controlled conditions. Withholding water at the tillering stage started the drought stress treatment. The soil water content reached $15 \%, 10 \%$, and $7.5 \%$ after 1 day, 3 days, and 4 days of drought treatment, respectively.

Yield performance was evaluated in experiments under drought stress and well-watered conditions at the experimental farm of International Rice Research Institute (Philippines). Seedlings of three genotypes were transplanted into a three-row plot with 45 plants per plot at a spacing of $15 \times 25 \mathrm{~cm}$ between rows and plants within each plot; three replications for each genotype. For drought stress treatment, water was drained and irrigation was held at the peak tillering stage until maturity. In the well-watered control, the field was irrigated at weekly intervals until 2 weeks before harvesting. Grain yield was measured on the 10 plants sampled at maturity from the middle row of each plot.

\section{SEM analysis}

The second leaves from 1-day drought-stressed plants grown in the pots were used for SEM analyses, as described 
in You et al. [68] with minor modifications. Fresh leaf samples were pre-fixed for $3 \mathrm{~h}$ in $3 \%$ glutaraldehyde-sodium phosphate buffer $(0.1 \mathrm{M})$ at room temperature and rinsed three times with $0.1 \mathrm{M}$ sodium phosphate buffer. Postfixation was performed with $2 \% \mathrm{OsO} 4$ at $4^{\circ} \mathrm{C}$. The samples were dehydrated through an ethanol series and infiltrated with an isoamyl acetate series. The samples were then coated with metal particles for analysis by SEM to observe the guard cells. A Hitachi S750 scanning electron microscope (http://www.hitachi-hitec.com/global/em/) was used to take photographs, and the numbers of guard cells in randomly chosen fields were counted and analyzed statistically.

\section{Physiological traits of the three genotypes under drought stress}

Detached leaves are weighed and saturated with water for $24 \mathrm{~h}$, then weighed again and dried for $48 \mathrm{~h}$, and weighed again. RWC was calculated using the following formula: RWC $(\%)=[(F M-D M) /(T M-D M)] \times 100$, where $F M$, $\mathrm{DM}$, and TM are the fresh, dry, and turgid masses of the tissue weighed, respectively. Monitoring the fresh weight loss at the indicated time points (per hour) measured the WLRs of detached leaves [68]. Measuring solute leakage from rice leaf tissue evaluated the REL, according to the method of Arora et al. [69], with minor modifications. Three replicates of $0.5 \mathrm{~g}$ fresh leaves were sampled from control and drought-treated plants. After being cut into 1$\mathrm{cm}$ pieces, the $0.5 \mathrm{~g}$ leaf samples were immersed in $20 \mathrm{~mL}$ distilled water in a test tube for $1 \mathrm{~h}$ with the help of a vacuum pump. After standing for $2 \mathrm{~h}$ at $25^{\circ} \mathrm{C}$, water conductivity was measured. Leaf discs were then killed in the same solution by autoclaving, and total conductivity was measured at room temperature. Percent injury arising from each treatment was calculated from the conductivity data using the equation: \% injury $=[(\% \mathrm{~L}(\mathrm{t})-\% \mathrm{~L}(\mathrm{c})) /$ $(100-\% \mathrm{~L}(\mathrm{c}))] \times 100)$, where $\% \mathrm{~L}(\mathrm{t})$ and $\% \mathrm{~L}(\mathrm{c})$ are percent conductivity for treated and control samples, respectively. Antioxidant enzyme activity, including catalase (CAT) and ascorbate peroxidase (APX), were determined following previously reported methods [70].

Enzyme-linked immuno-sorbent assay (ELISA) was performed to measure JA content. Three replicates of 200 $500 \mathrm{mg}$ fresh leaves were sampled from control and drought-stressed plants and immediately ground with $80 \%$ methanol under ice-bath conditions, endogenous JA content was measured according to the manufacture's protocol (RB Plant-JA, Cat No.DRE-P10695, USA).

\section{RNA extraction, RNA-seq library construction, and sequencing}

Three top leaves for each sample (two replicates for each sample) were harvested for each genotype under 1 day and 3 days of drought stress and under well-watered control conditions. All samples were immediately frozen in liquid nitrogen and stored at $-80^{\circ} \mathrm{C}$. The TRIzol Reagent (Invitrogen, USA) was used to extract total RNA, which was quantified by a Qubit RNA assay kit (Applied Biosystems, CA, USA). An Agilent 2100 Bioanalyzer (Agilent Technologies) was used to check the RNA integrity. The ribosomal RNA (rRNA) was removed from $8 \mu \mathrm{g}$ of total RNA using a RiboMinus ${ }^{\text {tix }}$ Plant Kit (Invitrogen), followed by a Ribo-Zero Gram-Negative Bacteria kit (Epicentre), according to the manufacturer's instructions. The TruSeq RNA Sample Preparation kit (Illumina) was used to construct the paired-end fragment library, with minor modifications. Briefly, the rRNA removed RNA was fragmented and the firststrand cDNA was synthesized using random hexamers and SuperScript II Reverse Transcriptase. The RNA template was then removed and a replacement strand was synthesized to generate double-stranded (ds) cDNA. After end repair and 3' end adenylation, an indexed adapter was ligated to the dsDNA. Fragments of 300-350 bp were excised and enriched by 12 cycles of PCR. The QUBIT and Agilent 2100 Bioanalyzer, assessed the yield and size distribution of the PCR products, respectively. CapitalBio Corporation, Beijing, China subjected the produced libraries to cluster generation on $\mathrm{cBot}$ and sequencing on a HiSeq 2000 platform (Illumina) with paired-end 100 base pair reads. The Illumina instrument software performed primary data analysis and base calling. Raw sequence data are available in the NCBI's Gene Expression Omnibus (GEO) database under the accession number GSE57950.

\section{Transcriptome data analysis}

An in-house perl script was used to remove adaptor sequences and low-quality sequences from the raw reads. The retained high-quality pair-end reads of rice for each sample were mapped to the rice genome of RGAP at MSU [71] by TopHat [72] and then assembled using Cufflinks [73] to construct unique transcripts sequences, using the parameter: -g -b - $\mathrm{u}-\mathrm{o}$. Cuffcompare [73] was used to compare the assembled transcript fragments of each sample to the reference annotation, constructing a nonredundant transcripts data set among the samples. The number of mapped clean reads for each gene was counted and normalized into the reads per kilo base per million value [74]; Cuffdiff [73] was then used to identify DEGs. Finally, genes with a $p$-value $\leq 0.001$ were designated as significantly differential expressed between each pair of samples.

Gene function annotations were performed based on the Rice Genome Annotation Project version 7 [71]. AgriGO was used to perform GO enrichment analysis [75]. The Kyoto encyclopedia of genes and genomes (KEGG) pathway enrichment was performed using a hypergeometric test. The analysis incorporated false discovery 
rate correction using the Benjamini and Hochberg method of multiple hypotheses testing to reduce false negatives [76]. A weighted gene co-expression network analysis [77] was used to construct gene coexpression networks based on a Pearson correlation coefficient $>0.75$.

\section{Quantitative real-time reverse transcription-PCR (qRT-PCR) analysis}

To validate the results of the Illumina sequencing experiment, an independent set of samples (three biological replicates per sample) were collected as described for the RNA sequencing. Total RNA was treated with DNase I (TransGene, Beijing, China) to remove residual genome DNA and cDNA synthesis was performed using EasyScript First-Strand cDNA Synthesis SuperMix (TransGene, Beijing, China) according to the manufacturer's protocol. qRT-PCR was used to verify a subset of DEGs, using the methods described by Swarbrick et al. [78]. The gene sequences were downloaded from the rice genome of RGAP at MSU [71]; Primer 3 software (http://frodo.wi.mit.edu/) was used to design the primers (Additional file 3). Twentyseven rice genes with various function categories were selected and tested in $20-\mu \mathrm{L}$ reactions using the SYBR ${ }^{\oplus}$ Green PCR Master Mix kit (Applied Biosystems, CA, USA), following the manufacturer's protocol, via an ABI Prism 7900 Sequence Detection System (Applied Biosystems). The relative expression of each gene was calculated according to the method of $2^{-\Delta \Delta \mathrm{Ct}}$ [79]. The Actin 1 gene (LOC_Os03g50890) was used as endogenous references for qRT-PCR, and all analyses were performed with three technical and three biological replicates.

\section{Additional files}

Additional file 1: Hierarchical cluster analysis of nine sample pools (columns) and all expressed genes, under control and drought stress conditions (rows). A PowerPoint file containing hierarchical cluster analysis of nine sample pools (columns) and all expressed genes, under control and drought stress conditions (rows). The raw data represented here can be obtained from GEO: GSE57950. In the colored panels, each horizontal line represents a single gene and the colored line indicates the expression level (in a log scale) of the gene relative to the median in a specific sample: high expression in red, low expression in green.

Additional file 2: Correlation analysis of Illumina sequencing results between two replicates of each sample for $\mathrm{H} 471, \mathrm{P} 28$, and $\mathrm{HHZ}$, under control (ck), 1 day and 3 days of drought stress, respectively. A PowerPoint file containing correlation analysis of Illumina sequencing results between two replicates of each sample for $\mathrm{H} 471, \mathrm{P} 28$, and $\mathrm{HHZ}$, under control (ck), 1 day and 3 days of drought stress, respectively.

Additional file 3: Information of primers used in qRT-PCR analysis. Excel file containing information of primers used in qRT-PCR analysis.

Additional file 4: Comparison of transcription measurements by Illumina sequencing and quantitative real-time reverse transcription-PCR (qRT-PCR) assays. A PowerPoint file containing comparison of transcription measurements by Illumina sequencing and quantitative real-time reverse transcription-PCR (qRT-PCR) assays. (A) The correlation coefficient $(R 2)$ between the two datasets is 0.93 . (B) Comparative analysis of six candidate genes expression level by qRT-PCR and RNA-seq.
qRT-PCR quantification values were compared with HHZ_ck. Error bars indicate the standard deviation. Actin 1 was used as an endogenous control.

Additional file 5: DEGs in P28 and H471 compared with $\mathrm{HHZ}$ under a well-watered condition. Excel file containing a list of DEGs in P28 and H471 compared with HHZ under a well-watered condition.

Additional file 6: GO enrichment analysis of DEGs in various groups. Excel file containing five lists of $\mathrm{GO}$ enrichment of DEGs in various groups: 6-1, GO enrichment analysis of genes with the higher basal expression in $\mathrm{H} 471$ compared with $\mathrm{HHZ} ; 6-2, \mathrm{GO}$ enrichment analysis of the commonly up-regulated genes in three genotypes under 1 day of drought stress; 6-3, GO enrichment analysis of the commonly down-regulated genes under 1 day of drought stress; 6-4, GO enrichment analysis of the commonly up-regulated genes in three genotypes under 3 days of drought stress; 6-5, $\mathrm{GO}$ enrichment of the commonly down-regulated genes in three genotypes under 3 days of drought stress (XLS $47 \mathrm{~kb}$ ).

Additional file 7: Genes list of higher basal expression in P28 and H471 compared with HHZ. Description: Excel file containing five list of genes with higher basal expression in P28 and $\mathrm{H} 471$ compared with $\mathrm{HHZ}$.

Additional file 8: DEGs list in $\mathrm{H} 471$ compared with $\mathrm{HHZ}$ under drought stress. Excel file containing a list of DEGs in H471 compared with $\mathrm{HHZ}$ under drought stress.

Additional file 9: List of DEGs with various function categories in H471 compared with $\mathrm{HHZ}$ under drought conditions. Excel file containing a list of DEGs with various function categories in $\mathrm{H} 471$ compared with $\mathrm{HHZ}$ under drought conditions.

Additional file 10: List of alpha-linolenic acid metabolism-related genes in $\mathbf{H 4 7 1}$ vs. HHZ DEGs. Excel file containing a list of alpha-linolenic acid metabolism-related genes in H471 vs. HHZ DEGs.

Additional file 11: Validation of JA related genes by qRT-PCR. A PowerPoint file containing comparison validation of eight JA related genes by qRT-PCR assay. qRT-PCR Quantification values were compared

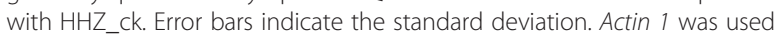
as an endogenous control.

Additional file 12: Information on introgressed chromosome segments and the differentially expressed genes in H471 compared with $\mathrm{HHZ}$ under control and drought stress conditions and their corresponding DT-related QTLs. Excel file containing information on introgressed chromosome segments and the differentially expressed genes in $\mathrm{H} 471$ compared with $\mathrm{HHZ}$ under control and drought stress conditions and their corresponding DT-related QTLS.

Competing interests

The authors declare that they have no competing interests.

Authors' contributions

$B F, Y Z$ and $Z L$ designed the experiments and drafted the manuscript. LH, FZ, $F Z$, and WW conducted the phenotype and RNA-seq experiments. FZ and WW performed the microarray data analysis, and FZ carried out the GRT-PCR confirmation of candidate genes. BF revised the manuscript. All authors read and approved the final manuscript.

\section{Acknowledgements}

This work was supported by the National 863 Project (2014AA106A603), the Bill \& Melinda Gates Foundation Project (OPP51587), and the Shenzhen Peacock Plan.

\section{Author details}

${ }^{1}$ Institute of Crop Sciences/National Key Facility for Crop Gene Resources and Genetic Improvement, Chinese Academy of Agricultural Sciences, South Zhong-Guan-Cun Street 12\#, Beijing 100081, China. ${ }^{2}$ Graduate School of Chinese Academy of Agricultural Sciences, South Zhong-Guan-Cun Street 12\#, Beijing 100081, China.

Received: 20 June 2014 Accepted: 30 October 2014

Published: 26 November 2014 
References

1. Farooq M, Wahid A, Kobayashi N, Fujita D, Basra SMA: Plant drought stress: effects, mechanisms and management. Agron Sustain Dev 2009, 29:185-212.

2. Lafitte HR, Li ZK, Vijayakumara CHM, Gao YM, Shi Y, Xu JL, Fu BY, Yu SB, Ali AJ, Domingo J, Maghirang R, Torres R, Mackill D: Improvement of rice drought tolerance through backcross breeding: evaluation of donors and selection in drought nurseries. Field Crops Res 2006, 97:77-86.

3. Hao W, Lin HX: Toward understanding genetic mechanisms of complex traits in rice. J Genet Genomics 2010, 37:653-666.

4. Millan $T$, Clarke H, Siddique K, Buhariwalla H, Gaur P, Kumar J, Gil J, Kahl G, Winter P: Chickpea molecular breeding: new tools and concepts. Euphytica 2006, 147:81-103.

5. Price AH, Cairns JE, Horton P, Jones HG, Griffiths H: Linking droughtresistance mechanisms to drought avoidance in upland rice using a QTL approach: progress and new opportunities to integrate stomatal and mesophyll responses. J Exp Bot 2002, 53:989-1004.

6. Krasensky J, Jonak C: Drought, salt, and temperature stress-induced metabolic rearrangements and regulatory networks. J Exp Bot 2012, 63:1593-1608.

7. Schroeder Jl, Allen GJ, Hugouvieux V, Kwak JM, Waner D: Guard cell signal transduction. Annu Rev Plant Physiol Plant Mol Biol 2001, 52:627-658.

8. Zhu JK: Salt and drought stress signal transduction in plants. Ann Rev Plant Biol 2002, 53:247-273.

9. Fan LM, Zhao Z, Assmann SM: Guard cells: a dynamic signaling model. Curr Opin Plant Biol 2004, 7:537-546.

10. McAinsh MR, Clayton H, Mansfield TA, Hetherington AM: Changes in stomatal behavior and guard cell cytosolic free calcium in response to oxidative stress. Plant Physiol 1996, 111:1031-1042

11. Pei ZM, Murata Y, Benning G, Thomine S, Klusener B, Allen GJ, Grill E, Schroeder Jl: Calcium channels activated by hydrogen peroxide mediate abscisic acid signalling in guard cells. Nature 2000, 406:731-734.

12. Kohler B, Hills A, Blatt MR: Control of guard cell ion channels by hydrogen peroxide and abscisic acid indicates their action through alternate signaling pathways. Plant Physiol 2003, 131:385-388.

13. Bright J, Desikan R, Hancock JT, Weir IS, Neill SJ: ABA induced NO generation and stomatal closure in Arabidopsis are dependent on $\mathrm{H}_{2} \mathrm{O}_{2}$ synthesis. Plant J 2006, 45:113-122.

14. Hamilton DW, Hills A, Kohler B, Blatt MR: $\mathrm{Ca}^{2+}$ channels at the plasma membrane of stomatal guard cells are activated by hyperpolarization and abscisic acid. Proc Natl Acad Sci U S A 2000, 97:4967-4972.

15. Creelman RA, Mullet JE: Jasmonic acid distribution and action in plants: regulation during development and response to biotic and abiotic stress. Proc Natl Acad Sci U S A 1995, 92:4114-4119.

16. Akter N, Sobahan MA, Uraji M, Ye WX, Hossain MA, Mori IC, Nakamura Y, Murata $Y$ : Effects of depletion of glutathione on abscisic acid and methyl jasmonate-induced stomatal closure in Arabidopsis thaliana. Biosci Biotechnol Biochem 2012, 76:2032-2037.

17. Daszkowska-Golec A, Szarejko I: Open or close the gate - stomata action under the control of phytohormones in drought stress conditions. Front Plant Sci 2013, doi:10.3389/fpls.2013.00138.

18. Phelix CF, Feltus FA: Plant stress biomarkers from biosimulations: the Transcriptome-To-Metabolome ${ }^{T M}\left(T M^{T M}\right)$ technology - effects of drought stress on rice. Plant Biol 2014, doi:10.1111/plb.12221.

19. Colebrook EH, Thomas SG, Phillips AL, Hedden P: The role of gibberellin signalling in plant responses to abiotic stress. J Exp Biol 2014, 217:67-75.

20. Shinozaki K, Yamaguchi-Shinozaki K: Gene networks involved in drought stress response and tolerance. J Exp Bot 2007, 58:221-227.

21. Zhou J, Wang X, Jiao Y, Qin Y, Liu X, He K, Chen C, Ma L, Wang J, Xiong L, Zhang Q, Fan L, Deng XW: Global genome expression analysis of rice in response to drought and high-salinity stresses in shoot, flag leaf, and panicle. Plant Mol Biol 2007, 63:591-608

22. Degenkolbe T, Do PT, Zuther E, Repsilber D, Walther D, Hincha DK, Kohl Kl: Expression profiling of rice cultivars differing in their tolerance to long-term drought stress. Plant Mol Biol 2009, 69:133-153.

23. Wang D, Pan YJ, Zhao XQ, Zhu LH, Fu BY, Li ZK: Genome-wide temporal-spatial gene expression profiling of drought responsiveness in rice. BMC Genomics 2011, 12:149.

24. Moumeni A, Satoh K, Kondoh H, Asano T, Hosaka A, Venuprasad R, Serraj R, Kumar A, Leung H, Kikuchi S: Comparative analysis of root transcriptome profiles of two pairs of drought-tolerant and susceptible rice near-isogenic lines under different drought stress. BMC Plant Biol 2011, 11:174
25. Gong Y, Rao LQ, Yu DQ: Abiotic Stress in Plants. In Chemistry "Agricultural Chemistry". Chapter 6. Edited by Stoytcheva M, Zlatev R. ; 2013. doi:10.5772/55865.

26. Li ZC, Mu P, Li CP, Zhang HL, Li ZK, Gao YM, Wang XK: QTL mapping of root traits in a doubled haploid population from a cross between upland and lowland japonica rice in three environments. Theor Appl Genet 2005, 110:1244-1252.

27. David TS, Henriques MO, Kurz-Besson C, Nunes J, Valente F, Vaz M, Pereira JS, Siegwolf R, Chaves MM, Gazarini LC, David JS: Water use strategies in two co-occurring Mediterranean evergreen oaks: surviving the summer drought. Tree Physiol 2007, 27:793-803.

28. Kadkhodaie A, Razmjoo J, Zahedi M: Peroxidase, Ascorbate Peroxidase and Catalase activities in Drought Sensitive, Intermediate and Resistance Sesame (Sesamum indicum L.) Genotypes. Int J Agron Plant Prod 2013, 4:3012-3021.

29. Yun KY, Park MR, Mohanty B, Herath V, Xu F, Mauleon R, Wijaya E, Bajic VB, Bruskiewich $R$, De los reyes $B G$ : Transcriptional regulatory network triggered by oxidative signals configures the early response mechanisms of japonica rice to chilling stress. BMC Plant Biol 2010, 10:16

30. Zhang T, Zhao XQ, Wang WS, Pan YJ, Huang LY, Liu XY, Zong Y, Zhu LH, Yang DC, Fu BY: Comparative transcriptome profiling of chilling stress responsiveness in two contrasting rice genotypes. PLoS One 2012, 7:e43274.

31. Zhang X, Byrnes JK, Gal TS, Li WH, Borevitz JO: Whole genome transcriptome polymorphisms in Arabidopsis thaliana. Genome Biol 2008 9:R165.

32. Jin P, Huang LY, Wang D, Wu HM, Zhu LH, Fu BY: Expression profiling of rice AP2/EREBP genes responsive to abiotic stresses. Sci Agr Sin 2009, 42:3765-3773.

33. Kim CY, Lee SH, Park HC, Bae CG, Cheong YH, Choi YJ, Han C, Lee SY, Lim CO, Cho MJ: Identification of rice blast fungal elicitor-responsive genes by differential display analysis. Mol Plant Microbe In 2000, 13:470-474.

34. Zhou YL, Xu MR, Zhao MF, Xie XW, Zhu LH, Fu BY, Li ZK: Genome-wide gene responses in a transgenic rice line carrying the maize resistance gene Rxo1 to the rice bacterial streak pathogen, Xanthomonas oryzae pv. oryzicola. BMC Genomics 2010, 11:78.

35. Liu FX, Xu WY, Wei Q, Zhang ZH, Xing Z, Tan LB, Di C, Yao DX, Wang CC, Tan YJ, Yan H, Ling Y, Sun CQ, Xue YB, Su Z: Gene expression profiles deciphering rice phenotypic variation between Nipponbare (japonica) and 93-11 (indica) during oxidative stress. PLoS One 2010, 5:e8632

36. Ross J, Li Y, Lim E, Bowles DJ: Higher plant glycosyltransferases. Genome Biol 2001, 2:reviews3004

37. Goel D, Singh AK, Yadav V, Babbar SB, Bansal KC: Overexpression of osmotin gene confers tolerance to salt and drought stresses in transgenic tomato (Solanum lycopersicum L.). Protoplasma 2010, 245:133-141.

38. Bell $E$, Mullet JE: Lipoxygenase gene expression is modulated in plants by water deficit, wounding, and methyl jasmonate. Mol Genet Genomics 1991, 230:456-462

39. Gardner HW: 9-Hydroxy-traumatin, a new metabolite of the lipoxygenase pathway. Lipids 1998, 33:745-749.

40. Sasaki A, Ashikari M, Ueguchi-Tanaka M, Itoh H, Nishimura A, Swapan D, Ishiyama K, Saito T, Kobayashi M, Khush GS, Kitano H, Matsuoka M: Green revolution: a mutant gibberellin-synthesis gene in rice. Nature 2002, 416:701-702.

41. Courtois B, McLaren G, Sinha PK, Prasad K, Yadav R, Shen L: Mapping QTLs associated with drought avoidance in upland rice. Mol Breed 2000, 6:55-66.

42. Lilley JM, Ludlow MM, McCouch SR, OToole JC: Locating QTL for osmotic adjustment and dehydration tolerance in rice. J Exp Bot 1996, 47:1427-1436.

43. Price AH, Townend J, Jones MP, Audebert A, Courtois B: Mapping QTLs associated with drought avoidance in upland rice grown in the Philippines and West Africa. Plant Mol Biol 2002, 48:683-695.

44. Wagner TA, Kohorn BD: Wall-associated kinases are expressed throughout plant development and are required for cell expansion. Plant Cell 2001, 13:303-318.

45. Osakabe Y, Yamaguchi-Shinozaki K, Shinozaki K, Tran LS: Sensing the environment: key roles of membrane-localized kinases in plant perception and response to abiotic stress. J Exp Bot 2013, 64:445-458.

46. Zamir D: Improving plant breeding with exotic genetic libraries. Nat Rev Genet 2001, 2:983-989.

47. Xu LX, Han LB, Huang BR: Antioxidant enzyme activities and gene expression patterns in leaves of kentucky bluegrass in response to 
drought and post-drought recovery. J Amer Soc Hort Sci 2011, 136:247-255.

48. Caldana C, Degenkolbe T, Cuadros-Inostroza A, Klie S, Sulpice R, Leisse A, Steinhauser D, Fernie AR, Willmitzer L, Hannah MA: High-density kinetic analysis of the metabolomic and transcriptomic response of Arabidopsis to eight environmental conditions. Plant J 2011, 67:869-884

49. Trzcinska-Danielewicz J, Bilsk A, Fronk J, Zielenkiewicz P, Jarochowsk E, Roszczyk M, Jończyk M, Axentowicz E, Skoneczny M, Sowiński P: Global analysis of gene expression in maize leaves treated with low temperature: I. Moderate chilling $\left(14^{\circ} \mathrm{C}\right)$. Plant Sci 2009, 177:648-658.

50. Koenig D, Jiménez-Gómez JM, Kimura S, Fulop D, Chitwood DH, Headland LR, Kumar R, Covington MF, Devisetty UK, Tat AV, Tohge T, Bolger A, Schneeberger K, Ossowski S, Lanz C, Xiong G, Taylor-Teeples M, Brady SM, Pauly M, Weigel D, Usadel B, Fernie AR, Peng J, Sinha NR, Maloof JN: Comparative transcriptomics reveals patterns of selection in domesticated and wild tomato. Proc Nat/ Acad Sci U S A 2013, 110:2655-2662.

51. Bell GD, Kane NC, Rieseberg LH, Adams KL: RNA-seq analysis of allelespecific expression, hybrid effects, and regulatory divergence in hybrids compared with their parents from natural populations. Genome Biol Evol 2013, 5:1309-1323.

52. Yasuda K, Tsukiyama T, Karki S, Okumoto Y, Teraishi M, Saito H, Tanisaka T: Mobilization of the active transposon $m$ Ping in interspecific hybrid rice between Oryza sativa and O. glaberrima. Euphytica 2013, 192:17-24.

53. Shivaprasad PV, Dunn RM, Santos BA, Bassett A, Baulcombe DC: Extraordinary transgressive phenotypes of hybrid tomato are influenced by epigenetics and small silencing RNAs. EMBO J 2012, 31:257-266.

54. Reddy AS, Ali GS, Celesnik H, Day IS: Coping with stresses: roles of calcium- and calcium/calmodulin-regulated gene expression. Plant Cell 2011, 23:2010-2032

55. Asano T, Hayashi N, Kikuchi S, Ohsugi R: CDPK-mediated abiotic stress signaling. Plant Signal Behav 2012, 7:817-821.

56. Peleg Z, Blumwald E: Hormone balance and abiotic stress tolerance in crop plants. Curr Opin Plant Biol 2011, 14:290-295.

57. Cheong JJ, Choi YD: Methyl jasmonate as a vital substance in plants. Trends Genet 2003, 19:409-413.

58. Harb A, Krishnan A, Ambavaram MM, Pereira A: Molecular and physiological analysis of drought stress in Arabidopsis reveals early responses leading to acclimation in plant growth. Plant Physiol 2010, 154:1254-1271.

59. Du H, Liu H, Xiong L: Endogenous auxin and jasmonic acid levels are differentially modulated by abiotic stresses in rice. Front Plant Sci 2013, 4:397.

60. Aimar D, Calafat M, Andrade AM, Carassay L, Abdala Gl, Molas ML: Drought tolerance and stress hormones: From model organisms to forage crops. In Plants and Environment. Edited by Dr. Hemanth Vasanthaiah. 2011. ISBN: 978-953-307-779-6, InTech, Available from: [http://www.intechopen.com/ books/plants-and-environment/drought-tolerance-and-stress-hormonesfrom-model-organisms-to-forage-crops]

61. Shan CJ, Liang ZS: Jasmonic acid regulates ascorbate and glutathione metabolism in Agropyron cristatum leaves under water stress. Plant Sci 2010, 178:130-139.

62. Wasternack C, Hause B: Jasmonates: biosynthesis, perception, signal transduction and action in plant stress response, growth and development. Ann Bot-london 2013, 111:1021-1058.

63. Lindemose S, O'Shea C, Jensen MK, Skriver K: Structure, function and networks of transcription factors involved in abiotic stress responses. Int J Mol Sci 2013, 14:5842-5878.

64. Rushton DL, Tripathi P, Rabara RC, Lin J, Ringler P, Boken AK, Langum TJ, Smidt L, Boomsma DD, Emme NJ, Chen X, Finer JJ, Shen QJ, Rushton PJ: WRKY transcription factors: key components in abscisic acid signalling. Plant Biotechnol J 2012, 10:2-11.

65. Nakashima K, Takasaki H, Mizoi J, Shinozaki K, Yamaguchi-Shinozaki K: NAC transcription factors in plant abiotic stress responses. Biochim Biophys Acta 1819, 2012:97-103.

66. Niu Y, Figueroa P, Browse J: Characterization of JAZ-interacting bHLH transcription factors that regulate jasmonate responses in Arabidopsis. $J$ Exp Bot 2011, 62:2143-2154.

67. Huang XH, Feng Q, Qian Q, Zhao Q, Wang L, Wang A, Guan JP, Fan DL, Weng QJ, Huang T, Dong GJ, Sang T, Han B: High-throughput genotyping by whole-genome resequencing. Genome Res 2009, 19:1068-1076.
68. You J, Zong W, Li XK, Ning J, Hu HH, Li XH, Xiao JH, Xiong LZ: The SNAC1targeted gene OsSRO1C modulates stomatal closure and oxidative stress tolerance by regulating hydrogen peroxide in rice. J Exp Bot 2013, 64:569-583.

69. Arora R, Pitchay DS, Bearce BC: Water-stress-induced heat tolerance in geranium leaf tissues: a possible linkage through stress proteins? Physiol Plant 1998, 103:24-34

70. Bonnecarre're V, Borsani O, Dı'az P, Capdevielle F, Blanco P, Monza J: Response to photoxidative stress induced by cold in japonica rice is genotype dependent. Plant Sci 2011, 180:726-732.

71. Kawahara Y, de la Bastide M, Hamilton JP, Kanamori H, McCombie WR, Ouyang S, Schwartz DC, Tanaka T, Wu J, Zhou S, Childs KL, Davidson RM, Lin H, Quesada-Ocampo L, Vaillancourt B, Sakai H, Lee SS, Kim J, Numa H, Itoh T, Buell CR, Matsumoto T: Improvement of the Oryza sativa Nipponbare reference genome using next generation sequence and optical map data. Rice 2013, 6:4

72. Trapnell C, Pachter $L$, Salzberg SL: TopHat: discovering splice junctions with RNA-Seq. Bioinformatics 2009, 25:1105-1111.

73. Trapnell C, Williams BA, Pertea G, Mortazavi A, Kwan G, van Baren MJ, Salzberg SL, Wold BJ, Pachter L: Transcript assembly and quantification by RNA-Seq reveals unannotated transcripts and isoform switching during cell differentiation. Nat Biotechnol 2010, 28:511-515.

74. Mortazavi A, Williams BA, McCue K, Schaeffer L, Wold B: Mapping and quantifying mammalian transcriptomes by RNA-Seq. Nat Methods 2008, 5:621-628.

75. Du Z, Zhou X, Ling Y, Zhang Z, Su Z: agriGO: a GO analysis toolkit for the agricultural community. Nucleic Acids Res 2010, 38:64-70.

76. Benjamini Y, Drai D, Elmer G, Kafkafi N, Golani I: Controlling the false discovery rate in behavior genetics research. Behav Brain Res 2001, 125:279-284.

77. Cao P, Jung KH, Choi D, Hwang D, Zhu J, Ronald PC: The rice oligonucleotide array database: an atlas of rice gene expression. Rice 2012, 5:17.

78. Swarbrick PJ, Huang K, Liu G, Slate J, Press MC, Scholes JD: Global patterns of gene expression in rice varieties undergoing a susceptible or resistant interaction with the parasitic Strigahermonthica. New Phytol 2008, 179:515-529.

79. Livak KJ, Schmittgen TD: Analysis of relative gene expression data using real-time quantitative PCR and the 2(-Delta Delta $C(T)$ ) Method. Methods 2001, 25:402-408.

doi:10.1186/1471-2164-15-1026

Cite this article as: Huang et al.: Comparative transcriptome sequencing of tolerant rice introgression line and its parents in response to drought stress. BMC Genomics 2014 15:1026.

\section{Submit your next manuscript to BioMed Central and take full advantage of:}

- Convenient online submission

- Thorough peer review

- No space constraints or color figure charges

- Immediate publication on acceptance

- Inclusion in PubMed, CAS, Scopus and Google Scholar

- Research which is freely available for redistribution 